\title{
Factores para la aceptación y uso de un sistema de gestión del aprendizaje en estudiantes de una universidad ecuatoriana
}

\author{
Factors for the Acceptance and Use of a Learning Management System in Students of an \\ Ecuadorian University
}

\section{Fatores para a aceitação e uso de um sistema de gestão da aprendizagem em estudantes de uma universidade equatoriana}

\author{
Juan Carlos Ocampo-Alvarado \\ Instituto Tecnológico y de Estudios Superiores de Monterrey \\ Monterrey, México \\ A00825409@itesm.mx \\ https://orcid.org/0000-0002-9353-7581 \\ María Paulina Ullauri-Ugarte \\ Instituto Tecnológico y de Estudios Superiores de Monterrey \\ Monterrey, México \\ A00825465@itesm.mx \\ https://orcid.org/0000-0002-9192-6348
}

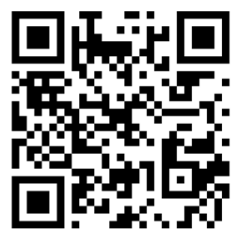

Recibido • Received • Recebido: 13 / 11 / 2019

Corregido • Revised • Revisado: 30 / 05 / 2021

Aceptado • Accepted • Aprovado: 25 / 08 / 2021

\begin{abstract}
Resumen:
Introducción: A pesar de la eficiencia comprobada de los sistemas de gestión de aprendizaje (SGA), su éxito dependerá del uso y aceptación del estudiantado. Por eso es imprescindible entender la interrelación de factores que determinan su utilización para diseñar estrategias, acciones e iniciativas efectivas que los promuevan. Objetivo. Analizar los factores para la aceptación y uso de un SGA en estudiantado universitario ecuatoriano. Metodología. La muestra final consistió en 304 estudiantes de cuatro carreras de una universidad privada, quienes llenaron un cuestionario diseñado con base en el modelo de aceptación tecnológica derivado de la teoría de la acción razonada. Se analizó la confiabilidad y validez del instrumento para luego emplear la técnica de modelos de ecuaciones estructurales por el método de mínimos cuadrados parciales (PLS-SEM). Resultados. El instrumento presentó una consistencia interna adecuada y una estructura tetrafactorial. Los resultados sugieren que la autoeficacia computacional influye positivamente en la facilidad de uso y utilidad percibida. Estas, a su vez, afectan la actitud respecto al uso, la cual influye directamente en el uso del sistema. Conclusión. El modelo de aceptación tecnológica es adecuado para estudiar los factores respecto
\end{abstract}


http://doi.org/10.15359/ree.25-3.10

http://www.una.ac.cr/educare

educare@una.ac.cr

al uso de los SGA. En promedio, el estudiantado percibe que el sistema es fácil de aprender y usar, pero representa poco beneficio. Dada la influencia de la autoeficacia computacional en el modelo propuesto, se recomienda tomar acciones para incrementar la exposición del alumnado a dispositivos tecnológicos y fomentar la automotivación por afianzar sus habilidades en estos.

Palabras clave: Ecuador; estudiante universitario; modelo de aceptación tecnológica; sistema de gestión de aprendizaje.

\begin{abstract}
:
Introduction. Despite the proven efficiency of Learning Management Systems (LMS), their success will depend on the use and acceptance of students. Therefore, it is essential to understand the relationship between the factors that determine their use in order to design effective strategies, actions, and initiatives that promote LMS usage. Objective. To analyze the factors that influence the acceptance and use of LMS in Ecuadorian university students. Method. The final sample consisted of 304 students from four different degree programs at a private university; the students filled out a questionnaire designed based on the Technological Acceptance Model derived from the theory of reasoned action. The reliability and validity of the instrument were analyzed, and then the Partial Least Squares structural equation modeling (PLS-SEM) was used. Results. The instrument showed an adequate internal consistency and a tetrafactorial structure. The results suggest that computational self-efficacy has a positive influence on ease of use and perceived utility. These, in turn, affect attitude towards use, which directly influences the use of the systems. Conclusion. The Technology Acceptance Model is suitable for studying factors regarding the use of LMS. On average, students perceive the systems to be easy to learn and use, but it represents little benefit. Given the influence of computational self-efficacy on the proposed model, we recommend taking action to increase student exposure to technological devices and encourage self-motivation by enhancing their skills in these.
\end{abstract}

Keywords: Ecuador; university student; technology acceptance model; learning management system.

\title{
Resumo:
}

Introdução. Apesar da eficiência comprovada dos Sistemas de Gestão da Aprendizagem (SGA), o seu sucesso dependerá do uso e aceitação dos seus estudantes. Portanto, é essencial compreender a inter-relação dos fatores que determinam a sua utilização, a fim de elaborar estratégias, ações e iniciativas eficazes que os promovam. Objetivo. Analisar os fatores que influenciam a aceitação e o uso de um SGA em estudantes universitários equatorianos. Metodologia. A amostra final consistiu de 304 estudantes de quatro carreiras de uma universidade particular, que preencheram um questionário desenhado com base no Modelo de Aceitação Tecnológica derivado da teoria da ação fundamentada. A confiabilidade e validade do instrumento foi analisada para depois usar a técnica de modelos da equação estrutural pelo método dos mínimos quadrados parciais (PLSSEM). Resultados. O instrumento apresentou uma consistência interna adequada e uma estrutura tetrafactorial. Os resultados sugerem que a autoeficácia computacional tem uma influência positiva na facilidade de uso e na percepção da utilidade. Estes, por sua vez, afetam a atitude relacionada ao uso, o que influencia diretamente no uso do sistema. Conclusão. O Modelo de aceitação tecnológica 
http://doi.org/10.15359/ree.25-3.10

\begin{abstract}
é adequado para o estudo de fatores relativos ao uso do SGA. Em média, os estudantes percebem que o sistema é fácil de aprender e usar, mas que representa pouco benefício. Dada a influência da autoeficácia computacional no modelo proposto, recomenda-se que sejam tomadas medidas para aumentar a exposição dos estudantes aos dispositivos tecnológicos, para encorajar a automotivação, melhorando as suas competências nesses dispositivos.
\end{abstract}

Palavras-chave: Equador; estudante universitário; modelo de aceitação tecnológica; sistema de gestão de aprendizagem.

\title{
Introducción
}

La tecnología es un aspecto fundamental de la educación y su efecto en el aprendizaje ha sido profusamente estudiado. Cada vez más instituciones de educación superior han adoptado herramientas digitales que posibilitan el uso de recursos tecnológicos como parte de la cátedra. Estas plataformas se conocen por muchos nombres: medio basado en internet, entorno virtual de aprendizaje o sistema de gestión de cursos (Bermúdez-Rodríguez y Fueyo-Gutiérrez, 2018). Sin embargo, en este trabajo se empleará sistema de gestión de aprendizaje (SGA).

Hay muchos SGA diferentes y todos comparten herramientas para crear contenido, administrar mecanismos de comunicación y gestionar evaluaciones (Arteaga Sánchez y Duarte Hueros, 2010). Entre ellos, los sistemas comerciales más utilizados provienen de Blackboard Corporation, mientras que los de código abierto más populares son Sakai, OLAT y Moodle. No obstante, la implementación de cualquiera de ellos implica una considerable inversión de recursos por parte de los centros educativos

A pesar de su eficiencia comprobada, su nivel de éxito dependerá en gran medida del uso y aceptación que obtenga del estudiantado (Arteaga Sánchez y Duarte Hueros, 2010). Establecer el SGA por sí mismo no proporciona beneficio alguno, si el estudiantado no lo utiliza. Por lo tanto, es imprescindible entender la compleja interrelación de factores que determinan dicho comportamiento, con el fin de diseñar estrategias, acciones e iniciativas efectivas que promuevan su uso.

Existen varios modelos para predecir la aceptación tecnológica. Uno de los más referidos es el modelo de aceptación tecnológica (TAM, por sus siglas en inglés) desarrollado por Davis (1989). Este se basa en la teoría de la acción razonada de Fishbein y Ajzen (Figura 1), la cual plantea un sistema general para comprender el comportamiento humano a partir de las creencias individuales (Hill, 1977). De acuerdo con la teoría, la creencia de que realizar una determinada conducta va a producir un resultado particular, mediatizado por la actitud hacia dicha conducta y las normas subjetivas, determina la intención que precede la acción en sí (Montaño y Kasprzyk, 2015). 
http://doi.org/10.15359/ree.25-3.10

http://www.una.ac.cr/educare

educare@una.ac.cr

Figura 1: Teoría de la Acción Razonada de Fishbein y Ajzen

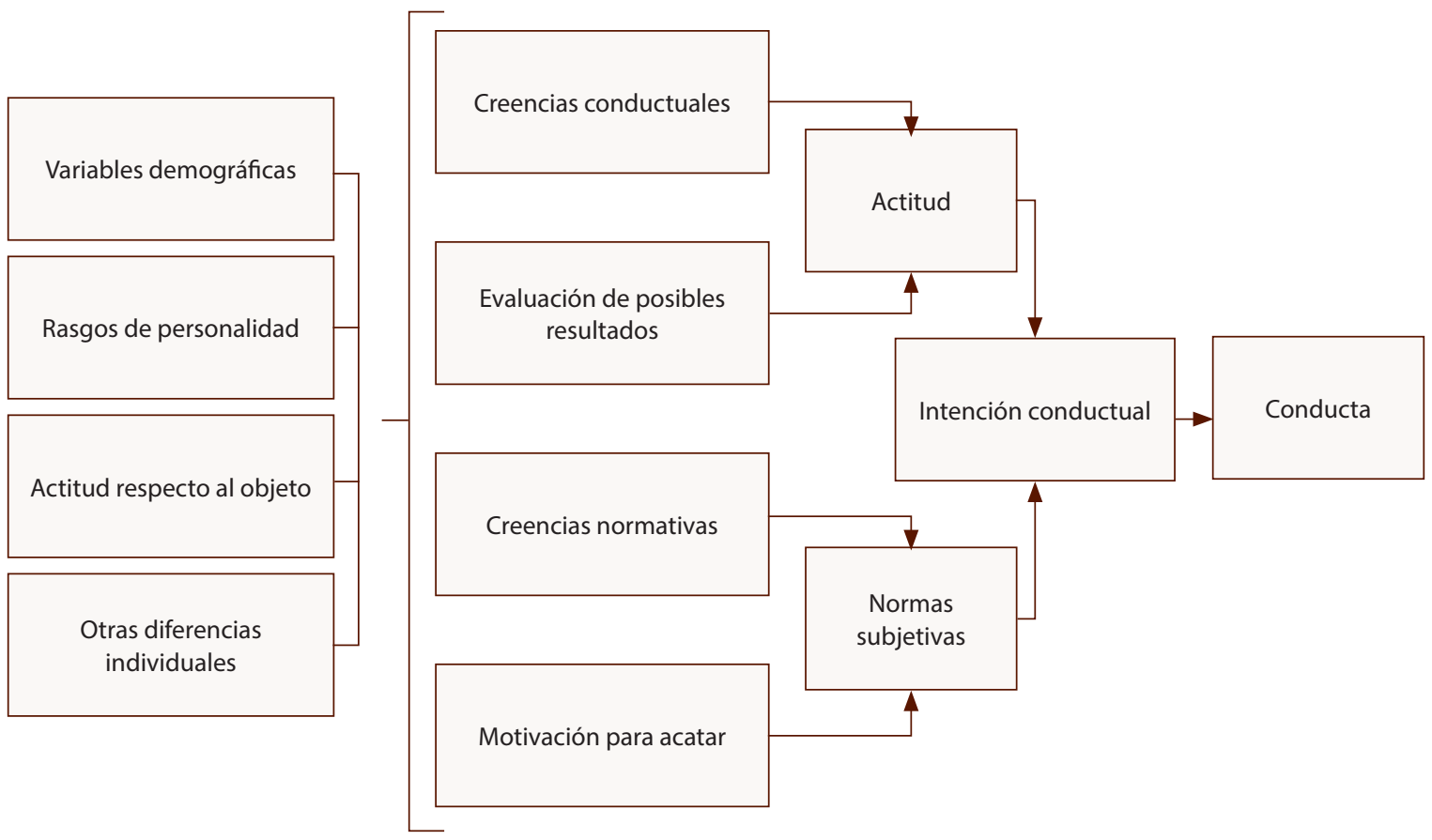

Nota: Elaboración propia, a partir de Montaño y Kasprzyk (2015).

Con base en dicho sistema, el TAM evalúa dos variables clave en la aceptación tecnológica, la utilidad percibida y la facilidad de uso. La primera es la valoración subjetiva que hace un individuo de que el uso de un sistema tecnológico podría mejorar su rendimiento en una acción específica y la segunda es el grado de esfuerzo que tendría que invertir para obtener un beneficio (Cabero-Almenara et al., 2018). Posteriormente, otras revisiones ahondaron en las innumerables variables externas que podrían influir en las creencias individuales, la actitud y el uso del sistema en sí (Govender y Rootman-le Grange, 2015).

Estos factores pueden dividirse en intrínsecos y extrínsecos. En el caso de los SGA, el modelo original ha sido expandido para incluir factores como la actitud de resistencia, conveniencia percibida, experiencia previa, esfuerzo esperado, condiciones facilitadoras, infraestructura tecnológica, entre otras (Cabero-Almenara et al., 2018). Pero como explican Sánchez Prieto et al. (2017), la adición de nuevos factores debe basarse en las circunstancias particulares del contexto estudiado con el fin, no solo de enriquecer el modelo y potenciar su poder explicativo, sino más bien de adaptarlo a cada caso. 
http://doi.org/10.15359/ree.25-3.10 http://www.una.ac.cr/educare educare@una.ac.cr

Una extensa revisión de la bibliografía muestra que el TAM ha sido ampliamente utilizado en el estudio de diversos sistemas tecnológicos incluidos los SGA (Tarhini et al., 2017). También ha sido replicado con éxito en muestras latinoamericanas (Cabero-Almenara et al., 2018; González-Bravo y Valdivia-Peralta, 2015; Samperio Pacheco y Barragán López, 2018). Con base en dicha revisión, se propone el siguiente modelo (Figura 2).

Figura 2: Modelo de investigación propuesto

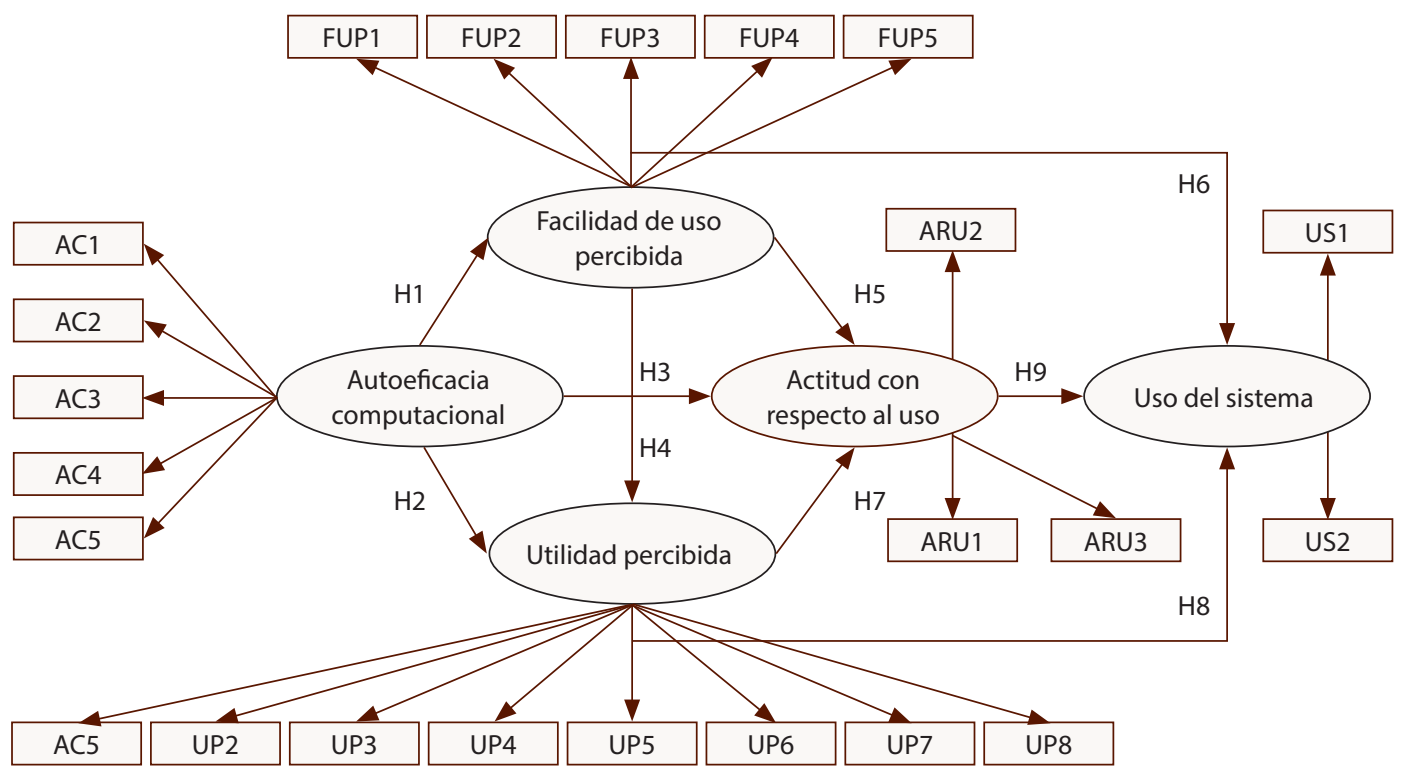

Nota: Elaboración propia.

Pese a la efectividad del TAM, son pocos los estudios revisados por pares sobre los factores motivacionales relacionados al uso de SGA en muestras estudiantiles ecuatorianas. Por ello, este estudio pretende analizar los factores que influyen en la aceptación de un SGA en una muestra de estudiantado universitario ecuatoriano, con el fin de suplir la carencia de investigación en este aspecto. Se espera que las conclusiones sirvan como lineamientos para ejecutar acciones que fomenten la aceptación y uso de los SGA.

\section{Método}

Con base en la clasificación de Ato et al. (2013), este estudio es selectivo, porque su característica distintiva es el empleo de la técnica del autoinforme para recopilar información empírica de las personas participantes; analítico, debido a que su objetivo es explicar el comportamiento, y transversal, debido a que se estudia una muestra en un único periodo de tiempo definido. 
http://doi.org/10.15359/ree.25-3.10

http://www.una.ac.cr/educare

educare@una.ac.cr

\section{Participantes}

Se empleó un muestreo por conveniencia, frecuente en estudios empíricos sobre aceptación tecnológica (Tarhini et al., 2017). Este permite al grupo investigador recopilar datos basados en la disponibilidad de la muestra. Pese a que se comparten las limitaciones propias del muestreo no probabilístico, los resultados de la investigación presente apuntan a una población diana con una problemática particular y, por lo tanto, es posible hacer uso de esta técnica (Etikan et al., 2016).

Participaron 309 estudiantes de la unidad curricular básica, correspondiente a los primeros tres semestres, de las carreras de Psicología Clínica, Psicología Organizacional, Comunicación Social y Pedagogía de la Universidad Católica de Santiago de Guayaquil (Tabla 1). Cinco fueron excluidos por incompletitud de respuestas. La muestra final consistió en 304 estudiantes entre 17 y 38 años ( $M=19.69 ; D T=2.57)$. El 73\% de la muestra es femenina con una edad promedio de $19,50$ años (DT $=2.51$ ) y el $23 \%$ es masculina con una edad promedio de 20,22 años (DT $=2,68)$. El 56,25\% pertenece a Psicología clínica. El 75,65\% utiliza Moodle en cuatro o más materias y el $68,75 \%$ lo hace semanalmente.

Tabla 1: Información demográfica de la muestra

\begin{tabular}{lrl}
\hline \multicolumn{1}{c}{ Variables } & $\mathrm{n}$ & \multicolumn{1}{l}{$(\%)$} \\
\hline Edad, M (DT) & 19.69 & $(2.57)$ \\
Género & 223 & $(73.35)$ \\
$\quad$ Femenino & 81 & $(26.65)$ \\
$\quad$ Masculino & & \\
Carrera & 171 & $(56.25)$ \\
$\quad$ Psicología clínica & 56 & $(18.42)$ \\
$\quad$ Comunicación social & 51 & $(16.77)$ \\
$\quad$ Psicología organizacional & 26 & $(8.55)$ \\
$\quad$ Educación & & \\
\# de materias en las que utilizan Moodle & 230 & $(75.65)$ \\
$\quad$ Cuatro o más & 38 & $(12.50)$ \\
$\quad$ Tres & 21 & $(6.90)$ \\
$\quad$ Dos & 15 & $(4.93)$ \\
$\quad$ Una & & \\
Frecuencia de uso de Moodle & 43 & $(14.14)$ \\
$\quad$ Mensual & 209 & $(68.75)$ \\
$\quad$ Semanal & 52 & $(17.10)$ \\
$\quad$ Diario
\end{tabular}

Nota: Elaboración propia 
http://doi.org/10.15359/ree.25-3.10

\section{Instrumento}

El instrumento está estructurado de la siguiente manera. La primera parte abarca la información demográfica de las personas participantes: género, edad, carrera, número de cursos en los que utiliza Moodle y frecuencia con la que usa dicho sistema. La segunda parte constituye el cuestionario basado en el TAM. Este fue diseñado por Venkatesh y Bala (2008) como revisión teórica del cuestionario propuesto por Venkatesh y Davis (2000). El cuestionario contiene veintitrés reactivos en escala de Likert de siete puntos desde "totalmente en desacuerdo" hasta "totalmente de acuerdo".

Los reactivos evalúan cinco factores: utilidad percibida (La plataforma Moodle me ayuda a aprender de forma más eficiente), autoeficacia computacional (Puedo acceder a los contenidos de la plataforma Moodle), facilidad de uso percibida (Usar la plataforma Moodle no representa esfuerzo alguno), actitud con respecto al uso (Usar la plataforma Moodle es una buena idea) y uso del sistema (Uso la plataforma Moodle en una escala de 1 (nunca) y 7 (siempre). Estos reactivos han sido empleados en múltiples investigaciones (Fathema et al., 2015; Govender y Rootman-le Grange, 2015; Solano Cóndor y Abella García, 2017; Tarhini et al., 2017).

\section{Procedimiento}

La recolección de datos fue realizada en sesiones colectivas dentro de los salones de clases y en el horario regular de cátedra. Además, se realizó durante los meses intermedios del curso académico para garantizar que incluso estudiantes de ingreso reciente estuvieran familiarizados con el SGA. Todas las personas participantes fueron informadas de i) el propósito de la investigación, ii) la voluntariedad de su participación y iii) del anonimato y confidencialidad de sus respuestas. La participación fue voluntaria y sin incentivo alguno. El tiempo de respuesta osciló entre 10 y 15 minutos, durante el cual al menos una persona encuestadora permaneció con las personas participantes para aclarar dudas.

\section{Análisis de datos}

Se utilizó IBM SPSS Statistics 20 y SmartPLS v3.2.7. Primero se evaluó la confiabilidad del instrumento empleando el coeficiente alfa de Cronbach, la correlación inter-reactivo y la correlación reactivo-total. A continuación, se realizó un análisis factorial exploratorio (AFE) para valorar la unidimensionalidad del instrumento y explorar su estructura factorial latente en aras de evaluar su validez. Después se llevó a cabo un análisis descriptivo de medias por factor para cotejarlas en función del género, empleando la prueba U de Mann-Whitney para corroborar la significación estadística.

Para examinar las hipótesis se usó el modelamiento de ecuaciones estructurales por mínimos cuadrados parciales. Este es un análisis de variables latentes que explora la dependencia de las relaciones multivariables que, en su modalidad de mínimos cuadrados parciales (PLS-SEM, 
http://doi.org/10.15359/ree.25-3.10

http://www.una.ac.cr/educare

educare@una.ac.cr

por sus siglas en inglés), es una aproximación de modelación causal que maximiza la varianza explicada por los constructos dependientes (Hair et al., 2012). Investigaciones pasadas del TAM han empleado esta técnica (Govender y Rootman-le Grange, 2015; Heflin et al., 2018; Samperio Pacheco y Barragán López, 2018).

\section{Modelo de investigación e hipótesis}

Se propuso un modelo que examina cuatro constructos (Figura 2). El primero es la autoeficacia computacional (AC), la cual da cuenta del grado en que el individuo cree tener la capacidad de realizar una tarea específica usando un dispositivo tecnológico (Venkatesh y Bala, 2008). Tiene un rol crucial modificando las actitudes que puede tener una persona con respecto a un sistema particular (Govender y Rootman-le Grange, 2015). Además, estudios previos han encontrado relaciones significativas entre esta y los factores originales (Venkatesh y Davis 1996; Venkatesh et al., 2000). Las siguientes hipótesis fueron planteadas:

$\mathrm{H}_{1}$. Autoeficacia computacional $(\mathrm{AC})$ tiene un efecto positivo en facilidad de uso percibida (FUP).

$\mathrm{H}_{2}$. Autoeficacia computacional (AC) tiene un efecto positivo en utilidad percibida (UP).

$\mathrm{H}_{3}$. Autoeficacia computacional (AC) tiene un efecto positivo en actitud con respecto al uso (ARU).

La influencia directa y positiva de la facilidad de uso percibida (FUP) en la utilidad percibida (UP) ha sido frecuentemente confirmada desde el origen del modelo (Davis, 1989; Venkatesh y Davis, 2000; Venkatesh et al., 2007; Venkatesh et al., 2000). De la misma manera, la FUP influye en la actitud con respecto al uso (ARU) y el uso del sistema (US) (Franco Castaño, 2016). Con base en lo mencionado, se proponen las siguientes hipótesis:

$\mathrm{H}_{4}$. Facilidad de uso percibida (FUP) tiene un efecto positivo en la utilidad percibida (UP).

$\mathrm{H}_{5}$. Facilidad de uso percibida (FUP) tiene un efecto positivo en actitud con respecto al uso (ARU).

$\mathrm{H}_{6}$. Facilidad de uso percibida (FUP) tiene un efecto positivo en el uso del sistema (US).

La bibliografía también indica que la UP es uno de los principales predictores de la intención de uso, por lo tanto, está estrechamente relacionada a la ARU (Davis 1989; Venkatesh y Davis, 2000). Además, Yong Varela (2004) plantea que existe una influencia significativa de esta variable con el US. Por lo tanto, se propone: 
http://doi.org/10.15359/ree.25-3.10

H7. La utilidad percibida (UP) tiene un efecto positivo en la actitud con respecto al uso (ARU).

H8. La utilidad percibida (UP) tiene un efecto positivo en el uso del sistema (US).

Por último, tanto el modelo original como sus revisiones concuerdan en que la actitud con respecto al uso tiene un efecto directo y positivo en el uso del sistema (Davis, 1989; Torres Albero et al., 2017; Venkatesh y Davis, 2000; Venkatesh et al., 2007; Venkatesh et al., 2000). Por ende, como última hipótesis se propone la siguiente.

H9. La actitud con respecto al uso (ARU) tiene un efecto positivo en el uso del sistema (US).

\section{Resultados y discusión}

Previo a la recolección de datos, el instrumento fue evaluado en dos fases: una revisión preliminar y una prueba piloto. El objetivo de la revisión preliminar fue optimizar el contenido de los reactivos y corregir posibles errores de inteligibilidad o ambigüedad en estos. Para esto se solicitó a cuatro profesionales del área de psicología, no pertenecientes al proyecto, examinar el cuestionario. Se realizaron leves ajustes para mejorar la claridad del instrumento, de acuerdo con las observaciones dadas.

A continuación, se realizó una prueba piloto con una muestra no aleatoria $(n=25)$ con características similares a las de la población diana, y se obtuvo una consistencia interna satisfactoria $(a>0.80)$. Esto permitió recoger in situ las reacciones de los encuestados para asegurar la completa comprensión del instrumento. Se examinaron los criterios de i) compresión de las instrucciones, ii) comprensión de los reactivos, iii) entendimiento del sistema de respuesta e iv) inteligibilidad de vocabulario. No se identificaron posibles errores de formato o contenido que puedan sesgar los resultados.

\section{Confiabilidad y validez del instrumento}

El cuestionario presentó un a de Cronbach de 0,942, lo cual indica una consistencia interna óptima dentro de los rangos convencionales y congruente con los hallazgos de estudios pasados en la región (Bedregal-Alpaca et al., 2019; Patiño-Toro et al., 2020). También presentó una media de correlación inter-reactivo de 0,433, que sugiere que estos son razonablemente homogéneos sin llegar a ser isomórficos (Piedmont, 2014). Finalmente, todos los reactivos reportaron correlaciones reactivo-total mayores a 0,350 ( $\mathrm{M}=0,623$; $\mathrm{DT}=0,08)$, o sea, ninguno es inconsistente con el comportamiento promedio del cuestionario (de Vaus, 2002). La Tabla 2 presenta estos resultados. 
http://doi.org/10.15359/ree.25-3.10

http://www.una.ac.cr/educare

educare@una.ac.cr

Tabla 2: Estadísticos descriptivos y de confiabilidad por reactivo

\begin{tabular}{|c|c|c|c|c|c|}
\hline$\#$ & Reactivo & M & DT & a & $r_{i-t}$ \\
\hline UP1 & La plataforma Moodle me ayuda a aprender de forma más eficiente & 3.73 & 1.74 & .939 & .630 \\
\hline UP2 & La plataforma Moodle permite mejorar mi rendimiento académico & 3.82 & 1.73 & .939 & 638 \\
\hline UP3 & La plataforma Moodle hace mi aprendizaje más dinámico & 3.88 & 1.71 & .940 & .614 \\
\hline UP4 & La plataforma Moodle facilita mi aprendizaje en la universidad & 4.09 & 1.73 & .939 & .631 \\
\hline UP5 & La plataforma Moodle me brinda mayor autonomía sobre mi aprendizaje & 4.43 & 1.75 & .939 & .667 \\
\hline UP6 & $\begin{array}{l}\text { Considero que la plataforma Moodle debería ser usada en todas y cada una } \\
\text { de las clases }\end{array}$ & 4.79 & 2.00 & .940 & .622 \\
\hline UP7 & Las ventajas de la plataforma Moodle sobrepasan las desventajas & 4.05 & 1.76 & .940 & 615 \\
\hline UP8 & En general, la plataforma Moodle es beneficiosa para mi aprendizaje & 4.27 & 1.73 & .938 & .739 \\
\hline $\mathrm{AC} 1$ & Puedo acceder a los contenidos de la plataforma Moodle & 5.05 & 1.82 & .940 & .602 \\
\hline $\mathrm{AC2}$ & Puedo navegar libremente por los contenidos de la plataforma Moodle & 5.07 & 1.77 & .939 & .650 \\
\hline AC3 & $\begin{array}{l}\text { Puedo usar la plataforma Moodle sin la necesidad de que me expliquen } \\
\text { cómo funciona }\end{array}$ & 5.02 & 1.88 & .940 & .599 \\
\hline AC4 & Puedo solucionar problemas que surgen de la plataforma Moodle & 3.98 & 1.90 & .941 & .555 \\
\hline AC5 & En general, puedo usar la plataforma Moodle & 5.37 & 1.73 & .939 & .632 \\
\hline FP1 & Usar la plataforma Moodle no representa esfuerzo alguno & 5.07 & 1.78 & .940 & .560 \\
\hline FP2 & Aprender a usar la plataforma Moodle fue fácil para mí & 5.49 & 1.65 & .940 & .615 \\
\hline FP3 & Es sencillo obtener información de la plataforma Moodle & 5.22 & 1.67 & .940 & 619 \\
\hline FP4 & El proceso de usar la plataforma Moodle es claro & 5.11 & 1.76 & .938 & .704 \\
\hline FP5 & En general, usar la plataforma Moodle es fácil & 5.30 & 1.72 & .939 & 657 \\
\hline ARU1 & Usar la plataforma Moodle es una buena idea & 5.07 & 1.87 & .938 & .715 \\
\hline ARU2 & La plataforma Moodle es una forma atractiva de aprender & 4.54 & 1.82 & .938 & .719 \\
\hline ARU3 & En general, me gusta usar la plataforma Moodle & 4.30 & 1.88 & .938 & .741 \\
\hline US1 & Uso la plataforma Moodle en una escala de 1 (nunca) y 7 (siempre) & 4.44 & 1.56 & .942 & .442 \\
\hline US2 & $\begin{array}{l}\text { El número de días a la semana que uso la plataforma Moodle en una escala de } \\
1 \text { (nada) y } 7 \text { (muchas) }\end{array}$ & 3.92 & 1.63 & .943 & .367 \\
\hline
\end{tabular}

M: media; DT: desviación típica; a: coeficiente alfa de Cronbach sin el reactivo; $r_{i-1}$ : correlación reactivo-total.

Nota: Elaboración propia. 
http://doi.org/10.15359/ree.25-3.10 http://www.una.ac.cr/educare educare@una.ac.cr

Dado que el instrumento pretende medir varios factores latentes, se optó por evaluar su unidimensionalidad. Tras confirmar la idoneidad de la muestra con el coeficiente de KaiserMeyer-Olkin (0.936) y la prueba de esfericidad de Bartlett $\left(X^{2}(253)=5627.67 ; p<.001\right)$, se realizó un análisis factorial exploratorio (AFE) por el método de componentes principales con rotación promax. Se encontraron cuatro factores con eigenvalores superiores a la unidad que explican el $70,66 \%$ de la varianza y corresponden a FUP-AC, UP, ARU y US (Tabla 3). Se rechaza la hipótesis concluyendo que el instrumento mide más de un factor y su estructura tetrafactorial concuerda con la construcción teórica de los reactivos.

Tabla 3: Análisis factorial exploratorio (rotación Promax) y varianza explicada

\begin{tabular}{ccccc}
\hline Variable & Factor 1 & Factor 2 & Factor 3 & Factor 4 \\
\hline UP1 & & .899 & & \\
UP2 & & .900 & & \\
UP3 & & .915 & & \\
UP4 & & .821 & & \\
UP5 & & .732 & & \\
UP6 & & .508 & & \\
UP7 & & .711 & & \\
UP8 & & .812 & & \\
AC1 & .743 & & & \\
AC2 & .768 & & & \\
AC3 & .785 & & & \\
AC4 & .634 & & & \\
AC5 & .790 & & & \\
FUP1 & .770 & & & \\
FUP2 & .813 & & & \\
FUP3 & .811 & & & \\
FUP4 & .875 & & & \\
FUP5 & .855 & & & \\
ARU1 & & & & \\
ARU2 & & & & \\
ARU3 & & & & \\
US1 & & & & \\
US2 & & & & \\
Varianza explicada (\%) & 44.453 & & \\
\hline
\end{tabular}

Método de extracción: Análisis de componentes principales; método de rotación: Promax con normalización de Kaiser.

Nota: Elaboración propia. 
http://doi.org/10.15359/ree.25-3.10

http://www.una.ac.cr/educare

educare@una.ac.cr

\section{Análisis descriptivo}

El reactivo con la mayor media fue FP2 ("Aprender a usar la plataforma Moodle fue fácil para mi"; $M=5.49 ; \mathrm{DT}=1.65$ ) y el reactivo con la menor fue UP1 ("La plataforma Moodle me ayuda a aprender de forma más eficiente"; $\mathrm{M}=3.73 ; \mathrm{DT}=1.74)$. Similarmente, el factor con la media más alta fue FUP ( $M=5.24$; DT = 1.71) y el factor con la más baja fue UP $(M=4.13$; $D T=1.77)$. Además, la asimetría y curtosis se mantuvieron en el rango de \pm 1 , ello sugiere que la muestra no se distribuye normalmente (Gravetter y Wallnau, 2014). Por último, los factores presentaron correlaciones significativas entre muy pobres y moderadas, según los criterios de Chan (2003). El estudiantado en promedio percibe que el sistema es fácil de aprender y utilizar; pero les representa poco beneficio o utilidad, lo cual afecta su actitud respecto a este. Así concuerda con trabajos pasados en que la facilidad de uso no antecede ni prevé la utilidad percibida, especialmente en sujetos nativos digitales (Urquidi et al., 2019).

Posteriormente, se analizaron las diferencias de medias en los factores según las submuestras por género. Considerando que los datos no siguen una distribución normal y las variables son del tipo ordinal, se empleó la prueba U de Mann-Whitney. Con base en los resultados de esta prueba, se rechaza la hipótesis nula y se reporta que los varones obtuvieron medias mayores que las mujeres en UP $(U=6900, p<.05), A C(U=7581, p<.05)$ y ARU $(U=7637$, $\mathrm{p}<.05$ ). Esto difiere con estudios previos que no han encontrado diferencias significativas por género (Al-Azawei et al. 2016; Cabero-Almenara et al., 2018). También contrasta con aquellos que encontraron diferencias significativas, con puntuaciones mayores en mujeres (González Aponcio y Calvo Aizpuru, 2018).

\section{Confiabilidad y validez del modelo}

Las cargas factoriales se utilizaron para evaluar la confiabilidad de los reactivos y el índice de fiabilidad compuesta (IFC) de los constructos. Todos los reactivos reportaron cargas factoriales adecuadas $(>0,70)$ a excepción de UP3 y AC4 que se mantuvieron por encima del valor crítico $(0,4)$, por lo que fueron conservados, mientras que el IFC de cada constructo $(>0,70)$ se mantuvo en los rangos óptimos (Hair et al., 2012).

La varianza media extraída se empleó para evaluar la validez convergente, alcanzando el valor recomendado $(>0,50)$ en todos los constructos (Hair et al., 2012). Para evaluar la validez discriminante se empleó el criterio de Fornell-Larcker, que sugiere que un constructo no debe compartir mayor varianza con otros que con sus variables asociadas, y el de cargas cruzadas, en el que cada reactivo debe presentar una asociación más fuerte con el constructo al cual fue teóricamente asignado que con los otros (Henseler et al., 2015). La Tabla 4 y 5 presenta los estadísticos de confiabilidad y validez del modelo. 
http://doi.org/10.15359/ree.25-3.10 http://www.una.ac.cr/educare educare@una.ac.cr

Tabla 4: Confiabilidad, validez convergente y validez discriminante por criterio de Fornell-Larcker

\begin{tabular}{lccccccccc}
\hline$\#$ & Constructo & IFC & AVE & $a$ & 1 & 2 & 3 & 4 & 5 \\
\hline 1 & UP & .928 & .618 & .928 & .786 & & & & \\
2 & AC & .859 & .550 & .856 & .533 & .742 & & & \\
3 & FUP & .923 & .708 & .922 & .392 & .859 & .841 & & \\
4 & ARU & .888 & .726 & .887 & .794 & .614 & .591 & .852 & \\
5 & US & .774 & .633 & .769 & .480 & .302 & .304 & .552 & .795 \\
\hline
\end{tabular}

IFC: índice de fiabilidad compuesta; AVE: varianza media extraída; $\alpha$ : coeficiente alfa de Cronbach *** $p<.001$

Nota: Elaboración propia.

Tabla 5: Cargas factoriales y validez discriminante por cargas cruzadas

\begin{tabular}{|c|c|c|c|c|c|c|c|}
\hline$\#$ & Constructo & Reactivo & 1 & 2 & 3 & 4 & 5 \\
\hline \multirow{8}{*}{1} & \multirow{8}{*}{ UP } & UP1 & .733 & .418 & .249 & .551 & .374 \\
\hline & & UP2 & .752 & .397 & .247 & .585 & .388 \\
\hline & & UP3 & .686 & .343 & .208 & .554 & .338 \\
\hline & & UP4 & .749 & .347 & .276 & .609 & .395 \\
\hline & & UP5 & .821 & .428 & .352 & .659 & .392 \\
\hline & & UP6 & .837 & .451 & .413 & .678 & .371 \\
\hline & & UP7 & .790 & .460 & .296 & .604 & .375 \\
\hline & & UP8 & .899 & .489 & .389 & .731 & .387 \\
\hline \multirow{5}{*}{2} & \multirow{5}{*}{$A C$} & $\mathrm{AC} 1$ & .396 & .721 & .608 & .449 & .185 \\
\hline & & $\mathrm{AC2}$ & .443 & .779 & .643 & .491 & .226 \\
\hline & & $\mathrm{AC} 3$ & .323 & .771 & .718 & .472 & .241 \\
\hline & & AC4 & .443 & .654 & .503 & .399 & .178 \\
\hline & & AC5 & .382 & .776 & .696 & .461 & .282 \\
\hline \multirow{5}{*}{3} & \multirow{5}{*}{ FUP } & FUP1 & .288 & .691 & .762 & .443 & .149 \\
\hline & & FUP2 & .302 & .683 & .808 & .477 & .301 \\
\hline & & FUP3 & .320 & .724 & .834 & .491 & .241 \\
\hline & & FUP4 & .396 & .785 & .933 & .552 & .288 \\
\hline & & FUP5 & .333 & .726 & .860 & .515 & .289 \\
\hline \multirow{3}{*}{4} & \multirow{3}{*}{ ARU } & ARU1 & .626 & .539 & .581 & .819 & .414 \\
\hline & & ARU2 & .703 & .486 & .452 & .855 & .477 \\
\hline & & ARU3 & .698 & .544 & .481 & .882 & .518 \\
\hline \multirow{2}{*}{5} & \multirow{2}{*}{ US } & US1 & .396 & .292 & .272 & .479 & .855 \\
\hline & & US2 & .368 & .181 & .208 & .397 & .731 \\
\hline
\end{tabular}

Resaltado indica la mayor carga factorial del reactivo. Nota: Elaboración propia. 
http://doi.org/10.15359/ree.25-3.10

http://www.una.ac.cr/educare

educare@una.ac.cr

\section{Evaluación del modelo de medida}

Como recomienda Hair et al. (2013), se empleó el bootstrapping con remuestreo de 5000 para probar el nivel de significancia de los coeficientes path dentro del modelo propuesto (Figura 3). $\mathrm{H}_{1}$ plantea que la AC tiene un efecto positivo en la FUP. El el coeficiente path y el estadístico $t$ presentan magnitudes adecuadas y son significativos $(\beta=0,859 ; t=29,649 ; \mathrm{p}<0,001)$. Por lo tanto, se admite $\mathrm{H}_{1}$. Igualmente, $\mathrm{H}_{2}$ plantea que la $A C$ tiene un efecto positivo en la UP y se admite bajo los mismos criterios $(\beta=0,747 ; t=4.236 ; \mathrm{p}<0.001)$. Por último, $\mathrm{H}_{3^{\prime}}$ la cual plantea que la $A C$ tiene un efecto positivo en la $A R U$, fue rechazada por superar el nivel de error máximo establecido $(\beta=-0,110 ; t=0,732 ; p>0,05)$.

Figura 3: Resultados del análisis de mínimos cuadrados parciales para el modelo propuesto

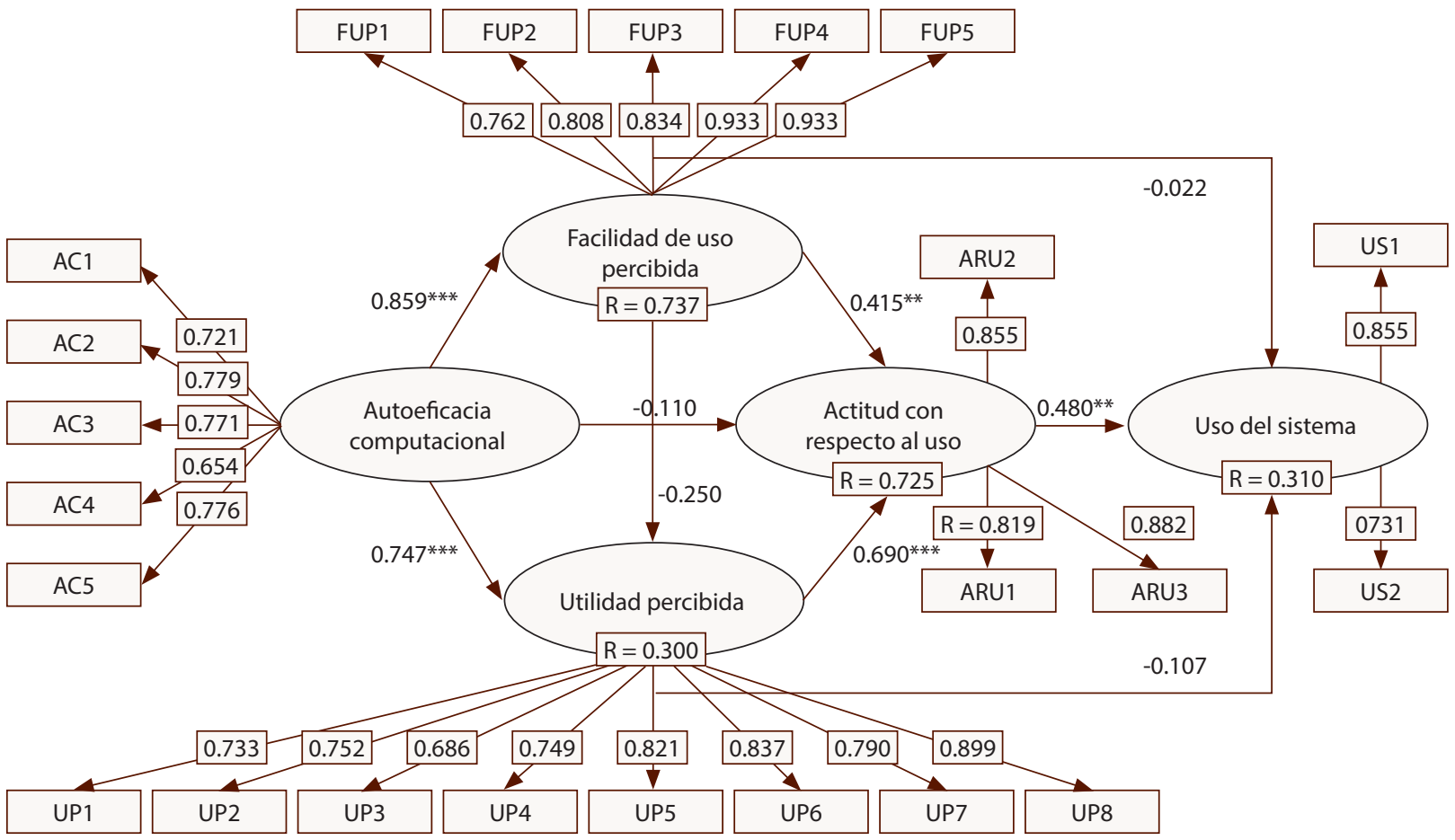

Nota: Elaboración propia.

A continuación, la $\mathrm{H}_{4}$ establece que la FUP tiene un efecto positivo en la UP, pero con base en los resultados obtenidos, es rechazada $(\beta=-0,250 ; t=1,373 ; \mathrm{p}>0,05)$. En cambio, la $\mathrm{H}_{5^{\prime}}$ que plantea la FUP tiene un efecto positivo en la ARU es confirmada $(\beta=0,415 ; t=3,194 ; p<$ 0,05). No obstante, la $\mathrm{H}_{6}$ que plantea que la FUP tiene un efecto positivo en la US también fue rechazada, con base en la magnitud de su coeficiente path, estadístico t y nivel de significancia $(\beta=-0,022 ; t=0,241 ; p>0,05)$. 
http://doi.org/10.15359/ree.25-3.10 http://www.una.ac.cr/educare educare@una.ac.cr

$\mathrm{H}_{7}$ plantea que la UP tiene un efecto positivo en la ARU y los resultados obtenidos soportan esta hipótesis $(\beta=0,690 ; t=11,691 ; \mathrm{p}<0,001)$. $\mathrm{La} \mathrm{H}_{8^{\prime}}$ en cambio, establece que la UP tiene un efecto positivo en el US, pero no alcanza el nivel de significancia necesaria y por lo tanto es rechazada $(\beta=0,107 ; t=0,831 ; p>0,05)$. Para finalizar, la $\mathrm{H}_{9}$ afirma que la ARU tiene un efecto positivo en el US y esta fue admitida tras el respectivo análisis de resultados, alcanza el nivel de significación adecuado $(\beta=0,480 ; t=3,271 ; \mathrm{p}<0,05)$. Así, las hipótesis aceptadas fueron $\mathrm{H}_{1^{\prime}} \mathrm{H}_{2^{\prime}}$ $\mathrm{H}_{5}, \mathrm{H}_{7}$ y $\mathrm{H}_{9}$, mientras que se rechazaron las hipótesis $\mathrm{H}_{3^{\prime}} \mathrm{H}_{4^{\prime}} \mathrm{H}_{6}$ y $\mathrm{H}_{8}$ (Tabla 6).

Tabla 6: Resultado de la prueba de hipótesis

\begin{tabular}{ccccccl}
\hline$\#$ & Hipótesis & Efecto & Coef. path & $t$ & $p$ & Resultados \\
\hline 1 & AC $(+) \rightarrow$ FUP & Directo & .859 & 29.649 & $* * *$ & Apoyada \\
2 & AC $(+) \rightarrow$ UP & Directo & .747 & 4.236 & $* * *$ & Apoyada \\
3 & AC $(+) \rightarrow$ ARU & Directo & -.110 & .732 & NS & No apoyada \\
4 & FUP $(+) \rightarrow$ UP & Directo & -.250 & 1.373 & NS & No apoyada \\
5 & FUP $(+) \rightarrow$ ARU & Directo & .415 & 3.194 & $* *$ & Apoyada \\
6 & FUP $(+) \rightarrow$ US & Directo & -.022 & .241 & NS & No apoyada \\
7 & UP $(+) \rightarrow$ ARU & Directo & .690 & 11.691 & $* * *$ & Apoyada \\
8 & UP $(+) \rightarrow$ US & Directo & .107 & .831 & NS & No apoyada \\
9 & ARU $(+) \rightarrow$ US & Directo & .480 & 3.271 & $* *$ & Apoyada \\
\hline
\end{tabular}

*** $p<.001$

${ }^{* *} \mathrm{p}<.01$

Nota: Elaboración propia.

Pese a las diferencias étnicas entre las muestras, estos hallazgos concuerdan con trabajos previos con PLS-SEM en donde el factor más influyente directa o indirectamente en la actitud respecto al uso de Moodle es la utilidad, seguido por la facilidad de uso (Al-Azawei et al. 2016; Calvo Porral, 2019; Rabaa'i, 2016). Otro estudio empleando PLS-SEM en una plataforma distinta encontró mayor influencia en los mismos dos factores: utilidad y facilidad (Yáñez-Luna y AriasOliva, 2018). En adelante la variabilidad de procedimientos de recolección de datos y métodos de análisis imposibilitan realizar comparaciones directas; no obstante, se esclarecen patrones transculturales que necesitan de mayor investigación. 
http://doi.org/10.15359/ree.25-3.10

http://www.una.ac.cr/educare

educare@una.ac.cr

\section{Conclusión}

Este estudio contribuye a explorar la interrelación de factores intrínsecos que afectan la aceptación y uso de un sistema tecnológico determinado en un centro académico. No obstante, puede ser aprovechado para trazar una línea base que permita el abordaje de temáticas afines en contextos similares. De forma general, se proporciona evidencia empírica encaminada a esclarecer los procesos de adopción tecnológica en estudiantado universitario para que instituciones, docentes y otras partes involucradas puedan orientar de forma más efectiva sus esfuerzos.

Las personas autoras de este artículo concuerdan con estudios pasados en que el modelo de aceptación tecnológica es adecuado para emplearse en investigaciones sobre el uso de los sistemas de gestión de aprendizaje. Este provee un marco referencial robusto que facilita la comprensión de la aceptación tecnológica, pero a su vez permite integrar nuevos factores de estudio, pudiendo ser adaptado a cada caso. Así se cuenta con una metodología probada e internacionalmente cotejable que también respeta y asume eficientemente las diferencias contextuales.

La correlación entre la facilidad de uso y autoeficacia computacional, así como su convergencia factorial, puede denotar la pericia tecnológica de los sujetos encuestados, un alto grado de facilidad del sistema o una combinación de estos. También puede sugerir que los esfuerzos institucionales de alfabetización sobre el uso de la plataforma son exitosos. Dada la influencia de la autoeficacia computacional, se recomienda tomar acciones dirigidas a incrementar la exposición del alumnado a dispositivos tecnológicos para fomentar la automotivación por afianzar sus habilidades. El estudiantado, ahora con mayor experticia, percibiría el sistema como más útil y amigable. Además, siendo un factor transversal en otras tecnologías, también contribuiría a la aceptación y uso de diferentes recursos de e-learning.

Considerando la baja utilidad percibida del sistema, se insta a las instituciones a determinar los principales beneficios de la plataforma para el estudiantado, alineando e integrando las necesidades y deseos de este, para configurar una propuesta de valor efectiva. Así, sería posible brindar un mensaje claro y consistente que modifique las preconcepciones del cuerpo estudiantil. De esta manera, se pretende que el estudiantado concluya razonablemente que utilizar la herramienta le genera valor.

Entre las limitaciones del estudio, se reconoce que se centra en un grupo poblacional muy concreto, por lo que la muestra es de carácter no-probabilístico y se asume que los resultados podrían no ser representativos. Por otro lado, es imprescindible analizar la confiabilidad y validez del instrumento a profundidad para determinar su adecuación al contexto latinoamericano. Finalmente, existen otros posibles factores que no fueron considerados dentro del modelo propuesto, tales como influencia social, motivación intrínseca, utilidad interpersonal y el contenido para el que cada cátedra emplea la plataforma. 
http://doi.org/10.15359/ree.25-3.10

Por esta razón, se sugiere que futuros estudios extiendan el modelo para asimilar más factores predominantes en la aceptación tecnológica. Uno de estos puede ser las estrategias individuales que cada docente utiliza para fomentar o coaccionar el uso de la plataforma por parte de sus estudiantes. Esto permitirá esclarecer cómo operan otras aristas que influyan en este proceso comportamental. En esta misma línea, se vuelve imprescindible estudiar los factores que influyen en la aceptación por parte del personal docente, reconociendo que son figuras clave en la implementación de cualquier iniciativa educativa.

\section{Declaración de Material complementario}

Este artículo tiene disponible, como material complementario:

-La versión preprint del artículo en https://doi.org/10.5281/zenodo.4412007

\section{Referencias}

Al-Azawei, A., Parslow, P. y Lundqvist, K. (2016). Investigating the effect of learning styles in a blended e-learning system: An extension of the technology acceptance model (TAM). Australasian Journal of Educational Technology, 33(2), 1-23. http://dx.doi.org/10.14742/ ajet.2758

Arteaga Sánchez, R. y Duarte Hueros, A. (2010). Análisis de las plataformas de enseñanza virtuales desde la perspectiva del TAM. En J. M. Pérez Tornero, J. Cabero Almenara y L. Vilches (Coords.), Congreso euro-iberoamericano de alfabetización mediática y culturas digitales (pp. 1-11). Universidad de Sevilla. https://idus.us.es/handle/11441/57007

Ato, M., López, J. J. y Benavente, A. (2013). Un sistema de clasificación de los diseños de investigación en psicología. Anales de Psicología, 29(3), 1038-1059. http://dx.doi. org/10.6018/analesps.29.3.178511

Bedregal-Alpaca, N., Cornejo-Aparicio, V., Tupacyupanqui-Jaén, D. y Flores-Silva, S. (2019). Evaluación de la percepción estudiantil en relación al uso de la plataforma Moodle desde la perspectiva del TAM. Ingeniare. Revista chilena de ingeniería, 27(4), 707-718. http://dx.doi. org/10.4067/S0718-33052019000400707

Bermúdez-Rodríguez, F. y Fueyo-Gutiérrez, M. A. (2018). Transformando la docencia: Usos de las plataformas de e-learning en la educación superior presencial. Revista Mediterránea de Comunicación, 9(2), 259-273. https://www.mediterranea-comunicacion.org/article/ view/2018-v9-n2-transformando-la-docencia-usos-de-las-plataformas-de-e-learning-enla-educacion-superior-presencial 
http://doi.org/10.15359/ree.25-3.10

http://www.una.ac.cr/educare

educare@una.ac.cr

Cabero-Almenara, J., Gallego Pérez, O., Puentes Puente, Á. y Jiménez Rosa, T. (2018). La "aceptación de la tecnología de la formación virtual" y su relación con la capacitación docente en docencia virtual. Edmetic Revista de Educación Mediática y TIC, 7(1), 225-241. https://doi.org/10.21071/edmetic.v7i1.10028

Calvo Porral, C. (2019). Aceptación de la plataforma Moodle por parte del alumnado universitario como herramienta de enseñanza-aprendizaje. En Red de Investigación e Innovación Educativa (Ed.), Conference proceedings 4 Virgtual International Conference on Education, Innovation and ICT (pp. 153-157). Redine. https://dialnet.unirioja.es/editor/13025

Chan, Y. H. (2003). Biostatistics 104: Correlational analysis. Singapore Medical Journal, 44(12), 614619. http://www.smj.org.sg/article/biostatistics-104-correlational-analysis

Davis, F.D. (1989). Perceived usefulness, perceived ease of use, and user acceptance of information technology. MIS Quarterly, 13(3), 319-340. https://doi.org/10.2307/249008

DeVaus, D. (2002).Analyzing socialscience data:50Key problems in data analysis. SAGEPublications. https://uk.sagepub.com/en-gb/eur/analyzing-social-science-data/book207458

Etikan, I., Abubakar Musa, S. y Sunusi Alkassim, R. (2016). Comparison of convenience sampling and purposive sampling. American Journal of Theoretical and Applied Statistics, 5(1), 1-4. https://doi.org/10.11648/j.ajtas.20160501.11

Fathema, N., Shannon, D. y Ross, M. (2015). Expanding the Technology Acceptance Model (TAM) to Examine Faculty Use of Learning Management Systems (LMSs) In Higher Education Institutions. MERLOT Journal of Online Learning and Teaching, 11(2), 210-232. https://jolt. merlot.org/Vol11no2/Fathema_0615.pdf

Franco Castaño, S. (2016). Factores determinantes de la aceptación tecnológica del e-commerce en los municipios del Altiplano del Oriente Antioqueño [Tesis de maestría]. Universidad Nacional de Colombia. https://repositorio.unal.edu.co/handle/unal/57658

González Aponcio, Z. y Calvo Aizpuru, M. (2018). Uso de la plataforma Moodle en el proceso de aprendizaje de las asignaturas de sistemas de información empresarial. En A. Vega Navarro y D. Stendardi (Coords.), De la innovación imaginada a los procesos de cambio (pp. 419434). Servicio de Publicaciones de la Universidad de la Laguna. https://riull.ull.es/xmlui/ handle/915/9444

González-Bravo, L.yValdivia-Peralta, M. (2015). Posibilidades para el uso del modelo de aceptación de la tecnología (TAM) y de la teoría de los marcos tecnológicos para evaluar la aceptación de nuevas tecnologías para el aseguramiento de la calidad en la educación superior chilena. Revista Electrónica Educare, 19(2), 181-196. http://dx.doi.org/10.15359/ree.19-2.11 
http://doi.org/10.15359/ree.25-3.10 http://www.una.ac.cr/educare educare@una.ac.cr

Govender, I. y Rootman-le Grange, I. (2015). Evaluating the early adoption of moodle at higher education institution. En A. Jeffries y M. Cubric (Eds.), 14th European conference on e-Learning (pp. 230-237). University of Hertfordshire. https://www.researchgate.net/ publication/320805629_Evaluating the early_adoption_of_Moodle_at_a_higher education institution

Gravetter, F. J. y Wallnau, L. B. (2014). Essentials of statistics for the behavioral sciences (8. ${ }^{\mathrm{a}}$ ed.). Wadsworth. https://www.cengagebrain.com.mx/shop/isbn/9781133956570

Hair, J. F., Jr., Hult, G. T. M., Ringle, C. M. y Sarstedt, M. (2013). A primer on partial least squares structural equation modeling (PLS-SEM). Sage Publications. https://us.sagepub.com/ en-us/nam/a-primer-on-partial-least-squares-structural-equation-modeling-pls-sem/ book244583

Hair, J. F., Sarstedt, M., Ringle, C. M. y Mena, J. A. (2012). An assessment of the use of partial least squares structural equation modeling in marketing research. Journal of the Academy of Marketing Science, 40(3), 414-433. https://doi.org/10.1007/s11747-011-0261-6

Heflin, H., Shewmaker, J. y Nguyen, J. (2018). Impact of mobile technology on student attitudes, engagement, and learning. Computers \& Education, 107, 91-99. https://doi.org/10.1016/j. compedu.2017.01.006

Henseler, J., Ringle, C. M. y Sarstedt, M. (2015). A new criterion for assessing discriminant validity in variance-based structural equation modeling. Journal of the Academy of Marketing Science, 43(1), 115-135. https://doi.org/10.1007/s11747-014-0403-8

Hill, R. J. (1977). [Trabajo de revisión del libro Belief, attitude, intention, and behavior: An introduction to theory and research, por M. Fishbein e I. Ajzen]. Contemporary Sociology, 6(2), 244-245. https://doi.org/10.2307/2065853

Montaño, D. E. y Kasprzyk, D. (2015). Chapter 6: Theory of reasoned action, theory of planned behavior. and the integrated behavioral model. En K. Glanz, B. Rimer y B. K. V. Viswanath (Eds.), Health behavior: Theory, research, and practice (5. ${ }^{a}$ ed., pp. 95-124). Jossey-Bass. https://psycnet.apa.org/record/2015-35837-000

Patiño-Toro, O. N., Bermeo-Giraldo, C., Valencia-Arias, A., Garcés-Giraldo, L. F. (2020). Factores que inciden en el aprendizaje en gestión tecnológica e innovación en estudiantes de administración mediante el modelo de aceptación tecnológica. Formación Universitaria, 13(5), 77-86. http://dx.doi.org/10.4067/S0718-50062020000500077

Piedmont, R. L. (2014). Inter-item correlations. En A. C. Michalos (Ed.), Encyclopedia of quality of life and well-being research (pp. 3303-3304). Springer. https://www.springer.com/gp/ book/9789400707528 
http://doi.org/10.15359/ree.25-3.10

http://www.una.ac.cr/educare

educare@una.ac.cr

Rabaa'i, A. A. (2016). Extending the technology acceptance Model (TAM) to assess students' behavioural intentions to adopt an elLearning system: The case of moodle as a learning tool. Journal of Emerging Trends in Engineering and Applied Sciences, 7(1), 13-30. https://www.researchgate.net/publication/325498824_Extending the_Technology Acceptance Model TAM to assess Students\%27 Behavioural Intentions to adopt an_e-Learning System_The Case_of_Moodle_as a Learning_Tool

Samperio Pacheco, V. M. y Barragán López, J. F. (2018). Análisis de la percepción de docentes, usuarios de una plataforma educativa a través de los modelos TPACK, SAMR y TAM3 en una institución de educación superior. Apertura: Revista de Innovación Educativa, 10(1), 116-131. https://www.ingentaconnect.com/content/doaj/16656180/2018/00000010/00 $\underline{000001 / a r t 00008}$

Sánchez Prieto, J. C., Olmos Migueláñez, S. y García-Peñalvo, F. J. (2017). ¿Utilizarán los futuros docentes las tecnologías móviles? Validación de una propuesta de modeloTAM extendido. RED. Revista de Educación a Distancia, 52(5), 1-30. https://doi.org/10.6018/red/52/5

Solano Cóndor, H. J. y Abella García, V. (2017). Factores determinantes de la aceptación de Cisco Netscape: Estudio empírico basado en TAM. Pixel-Bit: Revista de Medios y Educación, (51), 211-225. https://doi.org/10.12795/pixelbit.2017.i51.14

Tarhini, A., Hone, K., Liu, X.y Tarhini, T. (2017). Examining the moderating effect of individual-level cultural values on users' acceptance of E-learning in developing countries: Af structural equation modeling of an extended technology acceptance model. Interactive Learning Environments, 25(3), 306-328. https://doi.org/10.1080/10494820.2015.1122635

Torres Albero, C., Robles, J. M., De Marco, S. y Antino, M. (2017). Revisión analítica del modelo de aceptación de la tecnología. El cambio tecnológico. Papers: revista de sociología, 102(1) 5-27. https://doi.org/10.5565/rev/papers.2233

Urquidi Martín, A. C., Calabor Prieto, M. S. y Tamarit Aznar, C. (2019). Entornos virtuales de aprendizaje: Modelo ampliado de aceptación de la tecnología. Revista Electrónica de Investigación Educativa, 21, 1-12. https://doi.org/10.24320/redie.2019.21.e22.1866

Venkatesh, V. y Bala, H. (2008). Technology acceptance model 3 and a research agenda on interventions. Decision Sciences, 39(2), 273-315. https://doi.org/10.1111/j.15405915.2008.00192.x

Venkatesh, V.y Davis, F. (1996). A model of the antecedents of perceived ease of use:Development and test. Decision Sciences, 27(3), 451-481. https://doi.org/10.1111/j.1540-5915.1996. $\underline{\mathrm{tb} 00860 . \mathrm{x}}$ 
http://doi.org/10.15359/ree.25-3.10 http://www.una.ac.cr/educare educare@una.ac.cr

Venkatesh, V. y Davis, F. D. (2000). A theoretical extension of the technology acceptance model: Four longitudinal field studies. Management Science, 46(2), 186-204. https://doi. org/10.1287/mnsc.46.2.186.11926

Venkatesh, V., Davis, F. D. y Morris, M. G. (2007). Dead or Alive? The development, trajectory and future of technology adoption research. Journal of the Association for Information Systems, 8(4), 267-286. https://doi.org/10.17705/1jais.00120

Venkatesh, V., Morris, M. G. y Ackerman, P. L. (2000). A longitudinal field investigation of gender differences in individual technology adoption decision-making processes. Organizational Behavior and Human Decision Processes, 83(1), 33-60. https://doi.org/10.1006/ obhd.2000.2896

Yáñez-Luna, J. C. y Arias-Oliva, M. (2018). M-learning: Aceptación tecnológica de dispositivos móviles en la formación online. Revista Tecnología, Ciencia y Educación, (10), 13-34. https://doi.org/10.51302/tce.2018.193

Yong Varela, L. A. (2004). Modelo de aceptación tecnológica (TAM) para determinar los efectos de las dimensiones de cultura nacional en la acpetación de las TIC. Revista Internacional de Ciencias Sociales y Humanidades, SOCIOTAM, 14(1), 131-171. https://www.redalyc.org/ pdf/654/65414107.pdf 